\title{
On the equivalence of spherical splines with least-squares collocation and Stokes's formula for regional geoid computation
}

\author{
Vegard Ophaug $^{1}$ (D) Christian Gerlach ${ }^{2}$
}

Received: 5 October 2016 / Accepted: 19 April 2017 / Published online: 2 May 2017

(C) The Author(s) 2017. This article is an open access publication

\begin{abstract}
This work is an investigation of three methods for regional geoid computation: Stokes's formula, leastsquares collocation (LSC), and spherical radial base functions (RBFs) using the spline kernel (SK). It is a first attempt to compare the three methods theoretically and numerically in a unified framework. While Stokes integration and LSC may be regarded as classic methods for regional geoid computation, RBFs may still be regarded as a modern approach. All methods are theoretically equal when applied globally, and we therefore expect them to give comparable results in regional applications. However, it has been shown by de Min (Bull Géod 69:223-232, 1995. doi:10.1007/BF00806734) that the equivalence of Stokes's formula and LSC does not hold in regional applications without modifying the cross-covariance function. In order to make all methods comparable in regional applications, the corresponding modification has been introduced also in the SK. Ultimately, we present numerical examples comparing Stokes's formula, LSC, and SKs in a closed-loop environment using synthetic noise-free data, to verify their equivalence. All agree on the millimeter level.
\end{abstract}

Keywords Regional geoid computation - Stokes's formula · Least-squares collocation - Spherical radial base functions . Spline kernel

\section{Vegard Ophaug}

vegard.ophaug@nmbu.no

Christian Gerlach

gerlach@keg.badw.de

1 Faculty of Science and Technology, Norwegian University of Life Sciences (NMBU), Drøbakveien 31, 1433 Ås, Norway

2 Commission of Geodesy and Glaciology, Bavarian Academy of Sciences and Humanities, Alfons-Goppel Str. 11, 80539 Munich, Germany

\section{Introduction}

The global gravity field is typically represented using spherical harmonics (SH). Consequently, in regional gravity modeling, one usually splits the gravity signal into a global long-wavelength part which is modeled using $\mathrm{SH}$, and a regional short-wavelength part, which is modeled using a suitable regional method (Sansò and Sideris 2013).

There exist several methods for approximating Earth's regional gravity field, of which integral formula solutions to geodetic boundary value problems and least-squares techniques have emerged as common approaches (Nerem et al. 1995). A review and comparison of different regional gravity modeling concepts are given by Tscherning (1981). More recently, Schmidt et al. (2015) investigated different regional gravity modeling methods through an International Association of Geodesy Inter-Commission Committee on Theory Joint Study Group. Considering geoid computation in particular, Stokes's formula (Stokes 1849) and leastsquares collocation (LSC) (Krarup 1969; Moritz and Sünkel 1978; Moritz 1980) are treated in most geodetic text books (Heiskanen and Moritz 1967; Vaníček and Krakiwsky 1986; Hofmann-Wellenhof and Moritz 2006; Torge and Müller 2012).

Radial base functions (RBFs) are limited to a certain spatial region, making them suitable for regional gravity field modeling due to their space-localizing properties. There is a vast amount of RBFs to choose from, as long as they represent harmonic kernel functions. They are versatile in that their approximation characteristics and spatial distribution can be adjusted, making it possible to use them for all kinds of data sets and for combining different types of observations (e.g., Lieb et al. 2016). Regional gravity field modeling with RBFs can be done using numerical integration (e.g., Freeden and Schneider 1998; Schmidt et al. 2002; Liu and Sideris 
2003; Roland and Denker 2005) or least-squares estimation approaches (e.g., Schmidt et al. 2007; Lieb et al. 2016). In this work, we use the latter, which is the common geodetic approach, facilitating error analysis and propagation. The mathematical foundation of RBFs, the special RBFs known as spherical wavelets, and their application in multiscale analysis are given by, for example, Freeden et al. (1998), Schmidt (2001), Jekeli (2005), or Schmidt et al. (2007). In later years, we have observed an increased use of RBFs for regional gravity modeling (Roland 2005; Klees et al. 2008; Eicker 2008; Tenzer and Klees 2008; Wittwer 2009; Bentel 2013; Naeimi 2013; Bentel et al. 2013a, b; Eicker et al. 2014; Pock et al. 2012; Bucha et al. 2015, 2016; Naeimi et al. 2015; Farahani et al. 2016; Lieb et al. 2016).

In this work, we aim to show that regional geoid computation with RBFs is equivalent to Stokes's formula and LSC, in theory and in practice. Theoretical and numerical comparisons of Stokes's formula and LSC were done by de Min (1995), while a theoretical comparison of LSC and SKs was discussed by Eicker (2008), both of which we review and present in a unified framework. We show the theoretical equivalence of Stokes's formula, LSC, and RBFs in the global case, as well as the breakdown of the equivalence of Stokes's formula and LSC in regional applications. We introduce the remedial modification of the cross-covariance function of LSC also in the SKs, such that all methods are equal also in regional applications. Ultimately, we present numerical examples comparing the methods in a closed-loop environment, demonstrating their equivalence in practice.

Section 2 introduces the different modeling approaches, while their theoretical equivalence in the global case is shown in Sect. 3. The breakdown of their equivalence in regional applications is shown in Sect. 4, and the remedial modifications of LSC and SKs to restore their equivalence to Stokes's formula are applied. Numerical examples comparing the methods are given in Sect. 5, while Sect. 6 summarizes our results.

\section{Modeling approaches}

\subsection{Stokes's formula}

Geoid heights $N$ may be obtained from block mean gravity anomalies $\Delta \bar{g}$ by the integral formula of Stokes (1849). It globally integrates the gravity anomalies over the whole sphere $\sigma$, using the Stokes function $S$ as integration kernel (or weight),

$N(P)=\frac{R}{4 \pi \gamma} \iint_{\sigma} S\left(\psi_{P q}\right) \Delta \bar{g}_{q} \mathrm{~d} \sigma$, where $R$ is the spherical Earth radius, $\gamma$ is normal gravity evaluated on the surface of the reference ellipsoid, and $\psi_{P q}$ is the spherical distance between computation point $P$ and data point $q$. Equation (1) is a spherical convolution of the $\Delta \bar{g}_{q}$ function with the kernel $S\left(\psi_{P q}\right)$ and can be solved exactly by either numerical integration or by a one-dimensional fast Fourier transform (1D-FFT) (Haagmans et al. 1993), where the FFT is performed along parallels only. We have used the FFT approach, implemented according to

$N\left(\varphi_{P}\right)=\frac{R \Delta \varphi \Delta \lambda}{4 \pi \gamma} \mathscr{F}_{1}^{-1}\left\{\sum_{\varphi} \mathscr{F}_{1}\{S(\Delta \lambda)\} \cdot \mathscr{F}_{1}\left\{\Delta \bar{g}_{q} \cos \varphi\right\}\right\}$,

where $\mathscr{F}_{1}$ denotes the 1 D-FFT operator and $\mathscr{F}_{1}^{-1}$ its inverse, and $\Delta \varphi$ and $\Delta \lambda$ are the latitudinal and longitudinal spacing of the computation grid, respectively. Furthermore, the Stokes function is computed by its closed formula (HofmannWellenhof and Moritz 2006).

\subsection{Least-squares collocation}

LSC is an optimal estimation method in the statistical sense, allowing the estimation of arbitrary gravity field quantities from inhomogeneously distributed point observations (Moritz 1980). It takes advantage of the knowledge of the signal covariance and tries to minimize the prediction error.

Restricted to the case of geoid computation and in direct comparison with Eq. (1), LSC can be written as

$N(P)=\mathbf{C}_{P q}^{N g}\left(\mathbf{C}_{q q}^{g g}\right)^{-1} \Delta g_{q}$,

where $\mathbf{C}_{P q}^{\mathrm{Ng}}$ is a matrix containing the signal cross-covariances between the functionals $N$ and $\Delta g$ between computation point $P$ and observations $q$, and $\mathbf{C}_{q q}^{g g}$ is the auto-covariance matrix between all combinations of observations.

All covariances are obtained from the same covariance function $C(\psi)$, assumed to depend only on the horizontal distance $\psi$ between the considered points. In physical geodesy, we typically take the covariance function of the disturbing potential $T$ to be the basic covariance function, from which all covariances are computed by covariance propagation. The covariance function can be written as

$C^{T}\left(\psi_{P q}\right)=\frac{1}{8 \pi^{2}} \int_{\lambda=0}^{2 \pi} \int_{\theta=0}^{\pi} \int_{\alpha=0}^{2 \pi} T\left(\theta_{P}, \lambda_{P}\right) T\left(\theta_{q}, \lambda_{q}\right) \sin \theta \mathrm{d} \theta \mathrm{d} \lambda \mathrm{d} \alpha$,

where $(\theta, \lambda)$ are spherical coordinates, and the points $P(\theta, \lambda), q(\theta, \lambda)$ are on the surface of the unit sphere. 
The global integral in Eq. (4) can also be expressed as a Legendre series,

$C^{T}\left(\psi_{P q}\right)=\sum_{n=2}^{\infty} c_{n}^{T} P_{n}\left(\cos \psi_{P q}\right)$,

where $P_{n}\left(\cos \psi_{P q}\right)$ are the Legendre polynomials, and $c_{n}^{T}=$ $\left(\lambda_{n}^{T}\right)^{2} c_{n}$, with spectral eigenvalue (including dimensioning) $\lambda_{n}^{T}=G M / R$. The dimensionless signal degree variances $c_{n}$ can be computed from a set of spherical harmonic coefficients $\left\{\Delta \bar{C}_{n m}, \Delta \bar{S}_{n m}\right\}$,

$c_{n}=\sum_{m=0}^{n}\left(\Delta \bar{C}_{n m}^{2}+\Delta \bar{S}_{n m}^{2}\right)$,

as obtained from a global gravity model (GGM), or by different empirical degree-variance models (Tscherning and Rapp 1974; Jekeli 1978; Flury 2006; Rexer and Hirt 2015).

\subsection{Spherical splines}

RBFs are isotropic functions which store most of their energy in a limited spherical cap around their origin, i.e., they have a distinct space-localizing ability and are therefore said to have quasi-local support (Freeden et al. 1998).

Geoid computation with RBFs is performed according to

$N(P)=\sum_{k=1}^{K} \hat{d}_{k} B\left(\psi_{P k}\right)$,

where $B\left(\psi_{P k}\right)$ are the RBFs which are placed on spatially distributed grid points $k$, and $\hat{d}_{k}$ are point-specific gravity field parameters in the form of dimensionless coefficients.

As spherical isotropic functions, RBFs can be decomposed into a series of Legendre polynomials $P_{n}\left(\cos \psi_{P k}\right)$, where the spectral characteristics of a specific RBF depend on the choice of Legendre coefficients $B_{n}$ (see Eq. (8)). One choice of $B_{n}$ is to take the frequency response of the signal into account, characterized by the signal degree variances. These RBFs are known as spherical splines (Freeden et al. 1998; Jekeli 2005; Eicker 2008). In terms of a harmonic kernel, the spline kernel (SK) can be written as

$$
\begin{aligned}
B^{S K}\left(\psi_{P k}\right) & =\sum_{n=2}^{\infty} \frac{\sqrt{2 n+1}}{4 \pi R^{2}} \lambda_{n} \sigma_{n} P_{n}\left(\cos \psi_{P k}\right) \\
& =\sum_{n=2}^{\infty} \sum_{m=-n}^{n} \lambda_{n} B_{n}^{S K} Y_{n m}^{R}\left(\theta_{P}, \lambda_{P}\right) Y_{n m}^{R}\left(\theta_{k}, \lambda_{k}\right),
\end{aligned}
$$

where $\psi_{P k}$ is the spherical distance between computation point $P$ and the origin of the SK at grid point $k$, and $\lambda_{n}$ are the spectral eigenvalues including dimensioning of the considered functional (e.g., $\lambda_{n}$ is set to $\lambda_{n}^{N}$ in case of geoid computation). The Legendre coefficients are given by

$B_{n}^{S K}=\frac{\sigma_{n}}{\sqrt{2 n+1}}$,

where $\sigma_{n}=\sqrt{c_{n}}$ are the degree standard deviations. Furthermore,

$$
\begin{aligned}
Y_{n m}^{R}(\theta, \lambda)= & \frac{1}{R \sqrt{4 \pi}} \\
& \times\left\{\begin{array}{l}
\bar{P}_{n m}(\cos \theta) \cos m \lambda, \quad m=0,1,2, \ldots, n \\
\bar{P}_{n|m|}(\cos \theta) \sin |m| \lambda, \quad m=-n, \ldots,-2,-1
\end{array}\right.
\end{aligned}
$$

are the surface spherical harmonic base functions of degree $n$ and order $m$, as defined by Schmidt (2001). $\bar{P}_{n m}(\cos \theta)$ are the fully normalized associated Legendre functions.

We note that the Legendre coefficients of the spherical splines differ slightly from the ones of the covariance function, and this is due to norm convergence issues (Eicker 2008).

Equation (7) represents the synthesis of geoid heights from known coefficients $\hat{d}_{k}$. The coefficients are obtained from gravity field observations by integration or parameter estimation techniques. We follow the latter approach and write Eq. (7) in matrix notation as

$N(P)=\mathbf{A}^{N} \mathbf{d}$,

where $\mathbf{d}$ contains the spline coefficients $\hat{d}_{k}$ according to Eq. (7), and $\mathbf{A}^{N}$ represents the design matrix according to Eq. (8), with elements

$A_{P k}^{N}=\sum_{n=2}^{\infty} \sum_{m=-n}^{n} \lambda_{n}^{N} B_{n}^{S K} Y_{n m}^{R}\left(\theta_{P}, \lambda_{P}\right) Y_{n m}^{R}\left(\theta_{k}, \lambda_{k}\right)$,

and with $\lambda_{n}^{N}=G M /(R \gamma)$.

In agreement with geoid computation using Stokes's formula [Eq. (1)] or LSC [Eq. (3)], we derive the spline coefficients from observed gravity anomalies in an analysis step, by inversion of the linear model

$\Delta \mathbf{g}+\mathbf{v}=\mathbf{A}^{g} \mathbf{d}$,

where $\Delta \mathbf{g}$ is the observation vector and $\mathbf{v}$ is the error vector. $\mathbf{A}^{g}$ is the design matrix, with elements

$A_{k q}^{g}=\sum_{n=2}^{\infty} \sum_{m=-n}^{n} \lambda_{n}^{g} B_{n}^{S K} Y_{n m}^{R}\left(\theta_{k}, \lambda_{k}\right) Y_{n m}^{R}\left(\theta_{q}, \lambda_{q}\right)$.

and with $\lambda_{n}^{g}=G M / R^{2}(n-1)$. 
By inversion of the linear model [Eq. (13)], one coefficient for each SK needs to be determined. This is an ill-conditioned linear inverse problem, and the normal matrix must be regularized in order to solve the system. Numerous regularization techniques exist, and regularization remains an important topic within gravity field modeling with RBFs (Bentel et al. 2013b; Naeimi et al. 2015). We have applied Tikhonov regularization (Tikhonov 1963), where an extended norm which includes constraints on the unknowns is minimized. Thus, we have a damped least-squares problem where not only the parameters which best fit the observations are determined, but also the solution is kept smooth enough to allow a stable inversion. The smoothness constraint is set a priori and governed by the regularization matrix $\mathbf{R}$. The regularized solution can be written as

$\hat{d}_{k}=\left[\left(\mathbf{A}^{g}\right)^{T} \mathbf{A}^{g}+\alpha \mathbf{R}\right]^{-1}\left(\mathbf{A}^{g}\right)^{T} \Delta \mathbf{g}$,

where the regularization parameter $\alpha$ is a weight, balancing the contribution of observations and prior information to the solution. It is chosen as a compromise between a solution which is smoothed too much and significant parts of the signal is lost ( $\alpha$ too large), and a solution which is corrupted by highfrequency errors, and no physically meaningful solution is obtained ( $\alpha$ too small).

We determine the regularization parameter $\alpha$ by the Lcurve method proposed by Hansen and O'Leary (1993), as it has proven to be a suitable method for noise-free data (Bentel 2013). Regardless of the regularization method, an initial guess of the regularization parameter, $\alpha_{0}$, must be made. Here, we make an initial guess based on the condition number of the normal matrix and the maximum SH degree $N_{\max }$, i.e., $\alpha_{0}=8\|\mathbf{N}\| / N_{\max }^{3}$, where $\|\mathbf{N}\|$ is the norm of the normal matrix $\mathbf{N}=\mathbf{A}^{T} \mathbf{A}$ (Naeimi 2013).

In case of signal representation using SKs, $\mathbf{R}$ contains scalar products of the SKs located at different grid points (Eicker 2008). If the SKs would be orthogonal, $\mathbf{R}$ would become a diagonal matrix which could be represented by a scaled identity matrix. If the scaling factor is combined with $\alpha$, we can set $\mathbf{R}=\mathbf{I}$.

For bandlimited signals, as we employ them in discrete matrix computations, the SKs are not strictly orthogonal, and therefore, $\mathbf{R}$ is not a diagonal matrix (Eicker 2008). For non-bandlimited signals, orthogonality can theoretically be achieved, but at the cost of infinite energy along the main diagonal of R. Eicker (2008) discussed different modifications which restrict the elements along the main diagonal to finite values. Among them is the modification of the a priori signal covariance function, which is used to define the smoothness of the solution. This modification could possibly lead to our SK solution becoming inconsistent with LSC, because implicitly, two different covariance functions are used. The solutions of both methods would not represent the same gravity field.

Eicker (2008) compared different modified and unmodified solutions using either a fully occupied $\mathbf{R}$, or the approximation $\mathbf{R}=\mathbf{I}$, and found that the considerations regarding infinite energy and non-orthogonality are of rather theoretical significance. The best solutions were in fact achieved using the approximation $\mathbf{R}=\mathbf{I}$. Even though the numerical experiments of Eicker (2008) are related to downward continuation of satellite gravity data of relatively low spectral resolution, our own numerical examples (Sect. 5) indicate that this approximation also holds for higher spectral resolutions.

Ultimately, the regularized solution for the dimensionless spline coefficients is given by

$\hat{d}_{k}=\left[\left(\mathbf{A}^{g}\right)^{T} \mathbf{A}^{g}+\alpha \mathbf{I}\right]^{-1}\left(\mathbf{A}^{g}\right)^{T} \Delta \mathbf{g}$,

representing the analysis step.

\section{Global equivalence}

In the global case, Eqs. (1), (3), and (7) are equivalent gravity field representations. Furthermore, all three are equal to $\mathrm{SH}$, which is our point of departure.

The disturbing potential $T$ on Earth's surface is a harmonic function satisfying Laplace's equation. Its solution may be formulated as a spherical harmonic expansion,

$$
\begin{aligned}
T(P)= & \sum_{n=2}^{\infty} T_{n}(P)=\sum_{n=2}^{\infty} \lambda_{n}^{T} \\
& \times \sum_{m=0}^{n}\left[\Delta \bar{C}_{n m} \cos m \lambda+\Delta \bar{S}_{n m} \sin m \lambda\right] \bar{P}_{n m}(\cos \theta) .
\end{aligned}
$$

Stokes's formula [Eq. (1)] is the surface integral representation of the spherical harmonic expansion of $T$, subject to the boundary condition as given by the well-known spherical fundamental equation of physical geodesy,

$\Delta g=-\frac{\partial T}{\partial r}-\frac{2}{R} T$.

Taking the spherical harmonic expansion of $\Delta g$ and Eq. (18) into account, we can establish the relationship between $T$ and $\Delta g$ as

$T=\sum_{n=2}^{\infty} T_{n}=\sum_{n=2}^{\infty} \frac{R}{n-1} \Delta g_{n}$. 
Considering the orthogonality relations and addition theorem of $\mathrm{SH}, \Delta g_{n}$ is given by

$\Delta g_{n}=\frac{2 n+1}{4 \pi} \iint_{\sigma} \Delta g P_{n}(\cos \psi) \mathrm{d} \sigma$

such that Eqs. (19) and (20) in combination with Bruns's formula, $N=T \gamma^{-1}$, give Stokes's formula (cf. Eq. (1)),

$$
N(P)=\frac{R}{4 \pi \gamma} \iint_{\sigma}\left[\sum_{n=2}^{\infty} \frac{2 n+1}{n-1} P_{n}\left(\cos \psi_{P q}\right)\right] \Delta g_{q} \mathrm{~d} \sigma,
$$

where we identify the Stokes function expressed as a Legendre series,

$$
S\left(\psi_{P q}\right)=\sum_{n=2}^{\infty} \frac{2 n+1}{n-1} P_{n}\left(\cos \psi_{P q}\right) .
$$

Thus, Stokes's integral formula and SH are equivalent in the global case, which is an omen for the practical application of Eq. (1): As the integration is performed globally, globally available $\Delta g$ are needed.

Stokes's formula is equivalent to LSC, as the following review of the interpretation of de Min (1995) will show. Given an area of $i$ gravity anomalies $\Delta g_{i}$, we can predict gravity anomalies $\Delta g_{q}$ in any point $q$ on Earth, by means of LSC. Then, we can rewrite Eq. (1) as

$$
\begin{aligned}
N(P) & =\frac{R}{4 \pi \gamma} \iint_{\sigma} S\left(\psi_{P q}\right) \Delta g_{q} \mathrm{~d} \sigma \\
& =\frac{R}{4 \pi \gamma} \iint_{\sigma} S\left(\psi_{P q}\right)\left[\mathbf{C}_{q i}^{g g}\left(\mathbf{C}_{i j}^{g g}\right)^{-1} \Delta g_{i}\right] \mathrm{d} \sigma,
\end{aligned}
$$

and, because the gravity points $i$ do not depend on the integration element,

$$
N(P)=\frac{R}{4 \pi \gamma}\left[\iint_{\sigma} S\left(\psi_{P q}\right) \mathbf{C}_{q i}^{g g} \mathrm{~d} \sigma\right]\left(\mathbf{C}_{i j}^{g g}\right)^{-1} \Delta g_{i} .
$$

As the integration is performed globally, both functions inside of the integral can be written in terms of SH. The covariance function of $\Delta g$ is expressed as a Legendre series as follows,

$$
C^{g}\left(\psi_{P q}\right)=\sum_{n=2}^{\infty} c_{n}^{g} P_{n}\left(\cos \psi_{P q}\right)
$$

with $c_{n}^{g}=\left(\lambda_{n}^{g}\right)^{2} c_{n}$.
We insert Eqs. (22) and (25) into Eq. (24), and apply the decomposition theorem and orthogonality relations of $\mathrm{SH}$, to obtain

$$
\begin{aligned}
N(P)= & \frac{R}{4 \pi \gamma}\left[\iint_{\sigma} \sum_{n=2}^{\infty} \frac{2 n+1}{n-1} P_{n}\left(\cos \psi_{P q}\right)\right. \\
& \left.\times \sum_{n=2}^{\infty} c_{n}^{g} P_{n}\left(\cos \psi_{q i}\right) \mathrm{d} \sigma\right]\left(\mathbf{C}_{i j}^{g g}\right)^{-1} \Delta g_{i} \\
= & \sum_{n=2}^{\infty}\left[\frac{R}{\gamma(n-1)} c_{n}^{g} P_{n}\left(\cos \psi_{P i}\right)\right]\left(\mathbf{C}_{i j}^{g g}\right)^{-1} \Delta g_{i} \\
= & \mathbf{C}_{P i}^{N g}\left(\mathbf{C}_{i j}^{g g}\right)^{-1} \Delta g_{i},
\end{aligned}
$$

which is the LSC formula [Eq. (3)], and where we have used the relation $\gamma=G M / R^{2}$. As pointed out by de Min (1995), this derivation allows LSC to be interpreted as a two-step procedure. In the first step, least-squares prediction is performed continuously over the entire Earth, based on discrete point data. In the second step, global integration by Stokes's formula is performed on this continuous data set.

We now follow the interpretation of Eicker (2008) to show that the same prediction as in Eq. (3) can be done in RBF representation using SKs.

Omitting the regularization term $\alpha \mathbf{I}$ for simplicity, we insert the solution for the estimated spline parameters in Eq. (16) (analysis step) into Eq. (11) (synthesis step) and obtain

$N(P)=\mathbf{A}^{N}\left[\left(\mathbf{A}^{g}\right)^{T} \mathbf{A}^{g}\right]^{-1}\left(\mathbf{A}^{g}\right)^{T} \Delta \mathbf{g}$.

Using some matrix algebra, we can rearrange Eq. (27) as follows,

$N(P)=\mathbf{A}^{N}\left(\mathbf{A}^{g}\right)^{T}\left[\mathbf{A}^{g}\left(\mathbf{A}^{g}\right)^{T}\right]^{-1} \Delta \mathbf{g}$.

If we now compare Eqs. (3) and (28), we see that LSC and spline representation are identical if

$\mathbf{A}^{N}\left(\mathbf{A}^{g}\right)^{T}=\mathbf{C}_{P q}^{N g}$

and

$\mathbf{A}^{g}\left(\mathbf{A}^{g}\right)^{T}=\mathbf{C}_{q q}^{g g}$.

We now look at the matrix multiplications in Eqs. (29) and (30) in the limit case where the distribution of SKs becomes continuous. Suppose that we have sets of $K$ equidistant points $k$. In the limit, the sum will become the integral over the unit sphere, supposing an infinitely dense distribution of SKs. We get 


$$
\begin{aligned}
\mathbf{A}^{N}\left(\mathbf{A}^{g}\right)_{(P q)}^{T}= & \lim _{K \rightarrow \infty} \sum_{k=1}^{K} \\
& \times\left[\sum_{n=2}^{\infty} \sum_{m=-n}^{n} \lambda_{n}^{N} B_{n}^{S K} Y_{n m}^{R}\left(\theta_{P}, \lambda_{P}\right) Y_{n m}^{R}\left(\theta_{k}, \lambda_{k}\right)\right] \\
& \times\left[\sum_{\bar{n}=2}^{\infty} \sum_{\bar{m}=-\bar{n}}^{\bar{n}} \lambda_{\bar{n}}^{g} B_{\bar{n}}^{S K} Y_{\bar{n} \bar{m}}^{R}\left(\theta_{q}, \lambda_{q}\right) Y_{\bar{n} \bar{m}}^{R}\left(\theta_{k}, \lambda_{k}\right)\right] \\
= & \sum_{n=2}^{\infty} \sum_{m=-n}^{n} \sum_{\bar{n}=2}^{\infty} \sum_{\bar{m}=-\bar{n}}^{\bar{n}} \lambda_{n}^{N} \lambda_{n}^{g}\left(B_{n}^{S K}\right)^{2} \\
& \times Y_{n m}^{R}\left(\theta_{P}, \lambda_{P}\right) Y_{\bar{n} \bar{m}}^{R}\left(\theta_{q}, \lambda_{q}\right) \\
& \times \overbrace{\iint_{\sigma} Y_{n m}^{R}\left(\theta_{k}, \lambda_{k}\right) Y_{\bar{n} \bar{m}}^{R}\left(\theta_{k}, \lambda_{k}\right) \mathrm{d} \sigma}^{\infty} \\
= & \sum_{n=2}^{\infty} \lambda_{n}^{N} \lambda_{n}^{g} \frac{c_{n}}{2 n+1} \sum_{m=-n}^{n} Y_{n m}^{R}\left(\theta_{P}, \lambda_{P}\right) Y_{n m}^{R}\left(\theta_{q}, \lambda_{q}\right) \\
= & \sum_{n=2}^{\infty} \lambda_{n}^{N} \lambda_{n}^{g} c_{n} P_{n}\left(\cos \psi_{P q}\right)=\mathbf{C}_{P q}^{N g},
\end{aligned}
$$

and

$$
\begin{aligned}
\mathbf{A}^{g}\left(\mathbf{A}^{g}\right)_{(q q)}^{T}= & \lim _{K \rightarrow \infty} \sum_{k=1}^{K} \\
& \times\left[\sum_{n=2}^{\infty} \sum_{m=-n}^{n} \lambda_{n}^{g} B_{n}^{S K} Y_{n m}^{R}\left(\theta_{q}, \lambda_{q}\right) Y_{n m}^{R}\left(\theta_{k}, \lambda_{k}\right)\right] \\
& \times\left[\sum_{\bar{n}=2}^{\infty} \sum_{\bar{m}=-\bar{n}}^{\bar{n}} \lambda_{\bar{n}}^{g} B_{\bar{n}}^{S K} Y_{\bar{n} \bar{m}}^{R}\left(\theta_{q}, \lambda_{q}\right) Y_{\bar{n} \bar{m}}^{R}\left(\theta_{k}, \lambda_{k}\right)\right] \\
= & \sum_{n=2}^{\infty} \sum_{m=-n}^{n} \sum_{\bar{n}=2}^{\infty} \sum_{\bar{m}=-\bar{n}}^{n}\left(\lambda_{n}^{g}\right)^{2}\left(B_{n}^{S K}\right)^{2} \\
& \times Y_{n m}^{R}\left(\theta_{q}, \lambda_{q}\right) Y_{\bar{n} \bar{m}}^{R}\left(\theta_{q}, \lambda_{q}\right) \\
& \times \overbrace{\iint_{\sigma \bar{n}} Y_{n m}^{R}\left(\theta_{k}, \lambda_{k}\right) Y_{\bar{n} \bar{m}}^{R}\left(\theta_{k}, \lambda_{k}\right) \mathrm{d} \sigma}^{\infty} \\
= & \sum_{n=2}^{\infty}\left(\lambda_{n}^{g}\right)^{2} \frac{c_{n}}{2 n+1} \sum_{m=-n}^{n} Y_{n m}^{R}\left(\theta_{q}, \lambda_{q}\right) Y_{n m}^{R}\left(\theta_{q}, \lambda_{q}\right) \\
= & \sum_{n=2}^{\infty}\left(\lambda_{n}^{g}\right)^{2} c_{n} P_{n}\left(\cos \psi_{q q}\right)=\mathbf{C}_{q q}^{g g} .
\end{aligned}
$$

Thus, we are able to approximate the original covariance function by a finite sum of the similar harmonic SKs. With mathematical rigor, increasingly dense point distributions will never reach the continuous limit. Instead of the number of points, one may consider introducing area weights for points and investigate the limit case where these area weights approach zero. This issue was discussed by, for example, Rummel (1982) and later by Tscherning (1999). As we will see in Sect. 5, we do not need to reach the limit case in practice, as SKs and LSC tend to be identical already for moderate point densities. Therefore, we stick with the derivations in Eqs. (31) and (32), noting that in practical applications, the rigorous treatment of the surface integral does not deviate significantly from the point grid representation.

Finally, it may be shown that Tikhonov regularization and LSC are formally equivalent if we consider observation noise $\varepsilon$, giving $\overline{\mathbf{C}}_{q q}^{g g}=\mathbf{C}_{q q}^{g g}+\mathbf{C}_{\varepsilon \varepsilon}^{g g}$. Therefore, $\mathbf{C}_{\varepsilon \varepsilon}^{g g}=\sigma^{2} \mathbf{I}=\mathbf{R}$ is interpreted as the regularization matrix. Thus, LSC is equal to Tikhonov regularization with $\alpha=1$, and LSC may be considered a particular form of regularization with a statistical rationale for determining the regularization matrix. The interested reader is referred to Rummel et al. (1979) or Bouman (1998) for more on this topic.

\section{Regional applications}

As seen in Sect. 3, all methods are equivalent in the global case, and they can, in principle, be applied globally. This, however, is not practical due to the requirement of globally distributed high-resolution gravity data. In addition, global integration using Stokes's integral, the LSC formula applied to large data sets, as well as the global analysis of spline coefficients all require huge computational efforts.

Terrestrial gravity data of high resolution are not available globally, while GGMs have global coverage, but lack high resolution. Consequently, in practical regional geoid computation, both data sources are combined. Therefore, the long-wavelength part of the gravity signal is determined from a GGM and removed from the terrestrial data. In turn, regional geoid computation is applied to the residual gravity data in a limited area only. The modeling results are residual geoid heights, and the long-wavelength part of the GGM is restored to obtain the final geoid. This procedure is commonly referred to as the remove-restore technique (Denker 2013).

In the case of Stokes's formula, we consider available terrestrial gravity data in a spherical cap with radius $\psi_{0}$ around the computation point only. The spherical cap is usually called the inner zone. Typically, $\psi_{0}$ is chosen such that the resulting omission error is negligible, which depends on $N_{\max }$ of the GGM. Thus, Eq. (1) is only applied to the inner zone, and we get

$N(P)=\frac{R}{4 \pi \gamma} \iint_{\sigma} \bar{S}\left(\psi_{P q}\right) \Delta \bar{g}_{q} \mathrm{~d} \sigma$,

with

$\bar{S}\left(\psi_{P q}\right)=\left\{\begin{array}{ll}S\left(\psi_{P q}\right), & 0^{\circ} \leq \psi<\psi_{0} \\ 0, & \psi_{0} \leq \psi \leq \pi\end{array}\right.$. 
In its spectral form, $\bar{S}\left(\psi_{P q}\right)$ is written as (de Min 1995)

$\bar{S}\left(\psi_{P q}\right)=\sum_{n=2}^{\infty}\left[\frac{2}{n-1}-Q_{n}\left(\psi_{0}\right)\right] \frac{2 n+1}{2} P_{n}\left(\cos \psi_{P q}\right)$,

where $Q_{n}\left(\psi_{0}\right)$ are the Molodensky or truncation coefficients, which can be computed by the recurrence relation of Paul (1973).

Considering the two-step interpretation of LSC in Sect. 3, we see that if Eq. (3) is applied to residual data in the inner zone only, we also include the implicitly extrapolated gravity signal outside the inner zone. Thus, as demonstrated theoretically and numerically by de Min (1995), LSC and Stokes's formula are not equivalent in regional applications.

Following de Min (1995), we insert Eq. (35) into Eqs. (24) and (26) and obtain

$N(P)=\overline{\mathbf{C}}_{P i}^{N g}\left(\mathbf{C}_{i j}^{g g}\right)^{-1} \Delta g_{i}$,

with

$\overline{\mathbf{C}}_{P i}^{N g}=\sum_{n=2}^{\infty} \frac{R}{2 \gamma}\left[\frac{2}{n-1}-Q_{n}\left(\psi_{0}\right)\right] c_{n}^{g} P_{n}\left(\cos \psi_{P i}\right)$.

Therefore, in terms of the two-step interpretation, leastsquares prediction is still performed continuously over the entire Earth, but Stokes's formula is applied in the inner zone only. Thus, LSC is again identical with Stokes's formula.

Equation (35) may be rewritten as follows,

$\bar{S}\left(\psi_{P q}\right)=\sum_{n=2}^{\infty}\left[1-\frac{n-1}{2} Q_{n}\left(\psi_{0}\right)\right] \frac{2 n+1}{n-1} P_{n}\left(\cos \psi_{P q}\right)$,

where we, in comparison with Eq. (22), isolate the modification of the Stokes function in brackets. Consequently, considering the global equivalence of LSC and SKs (Sect. 3), the modification must be introduced in the SKs in the synthesis step [Eq. (11)]. By introducing the Molodensky coefficients in Eq. (11), the SKs get strict local support (Kusche 2002). Then, the elements of $\mathbf{A}^{N}$ are given as follows,

$$
\begin{aligned}
A_{P k}^{N}= & \sum_{n=2}^{\infty} \sum_{m=-n}^{n} \lambda_{n}^{N}\left[1-\frac{n-1}{2} Q_{n}\left(\psi_{0}\right)\right] \\
& \times B_{n}^{S K} Y_{n m}^{R}\left(\theta_{P}, \lambda_{P}\right) Y_{n m}^{R}\left(\theta_{k}, \lambda_{k}\right) .
\end{aligned}
$$

The unmodified and modified cross-covariance functions and SKs are shown in Fig. 1, based upon the computation scheme of the numerical examples in Sect. 5. The general function characteristics do not change much, as we deal with regional applications and have restricted the computations to the spectral band above degree 250. By doing this, the contribution from the gravity signal outside the inner zone is relatively small, i.e., our problem is already localized to some extent. However, we note that the modified functions tend to show a larger degree of localization than the unmodified functions. Furthermore, modification of the SK generates a saddle point around spherical distances close to the inner zone cap radius $\psi_{0}=1^{\circ}$. Compared to the modified SK, this feature is less prominent in the modified cross-covariance function, because the Molodensky coefficients contribute to a lesser extent in the cross-covariance function than they do in the SK. In both cases, the $Q_{n}$ enter in linear form, but while the SK is also a linear quantity [units of m, see Eq. (39)], the cross-covariance function is quadratic [units of $\mathrm{m} \cdot \mathrm{mGal}$, see Eq. (37)] and thus less affected by the linear contribution of the $Q_{n}$.

\section{Numerical examples}

Here we aim to demonstrate to what extent the different approaches are equivalent in practical geoid computation. To do this, we perform a comparative assessment of SKs, LSC, and Stokes's formula in a closed-loop environment using synthetic data, see Fig. 2. Both noise-free synthetic observations (in the form of gravity anomalies $\Delta g$ ) and the true validation geoid are computed by spherical harmonic synthesis (SHS) using the EGM2008 GGM (Pavlis et al. 2012), with $251 \leq n \leq 2190$, to simulate the remove-restore technique (where we assume that the long-wavelength part of the gravity signal has been removed using a typical GOCE-based GGM of $N_{\max }=250$ ). Thus, the residual gravity anomalies are computed as follows,

$$
\begin{aligned}
\Delta g(P)= & \sum_{n=251}^{2190} \lambda_{n}^{g} \sum_{m=0}^{n}\left[\Delta \bar{C}_{n m} \cos m \lambda+\Delta \bar{S}_{n m} \sin m \lambda\right] \\
& \times \bar{P}_{n m}(\cos \theta) .
\end{aligned}
$$

Geoid heights by Stokes's formula, LSC, and SKs are compared in terms of RMS differences to the validation geoid computed by SHS (to which they should all be equivalent). By this approach, we not only validate the internal consistency of the methods, but also check whether the methods are correct in the absolute sense by comparison with an external reference. However, in order to make the $\mathrm{SH}$ validation geoid equivalent to geoids computed by the regional methods, where a spherical cap with radius $\psi_{0}$ is considered, the truncation coefficients (Sect. 4) are also introduced in the SHS as follows, 
Fig. 1 Unmodified and modified a cross-covariance functions and $\mathbf{b}$ SKs, based on the computation scheme of the numerical examples in Sect. 5. Note that

$C^{N g}=\bar{C}^{N g}\left(\psi_{0}=180^{\circ}\right)$
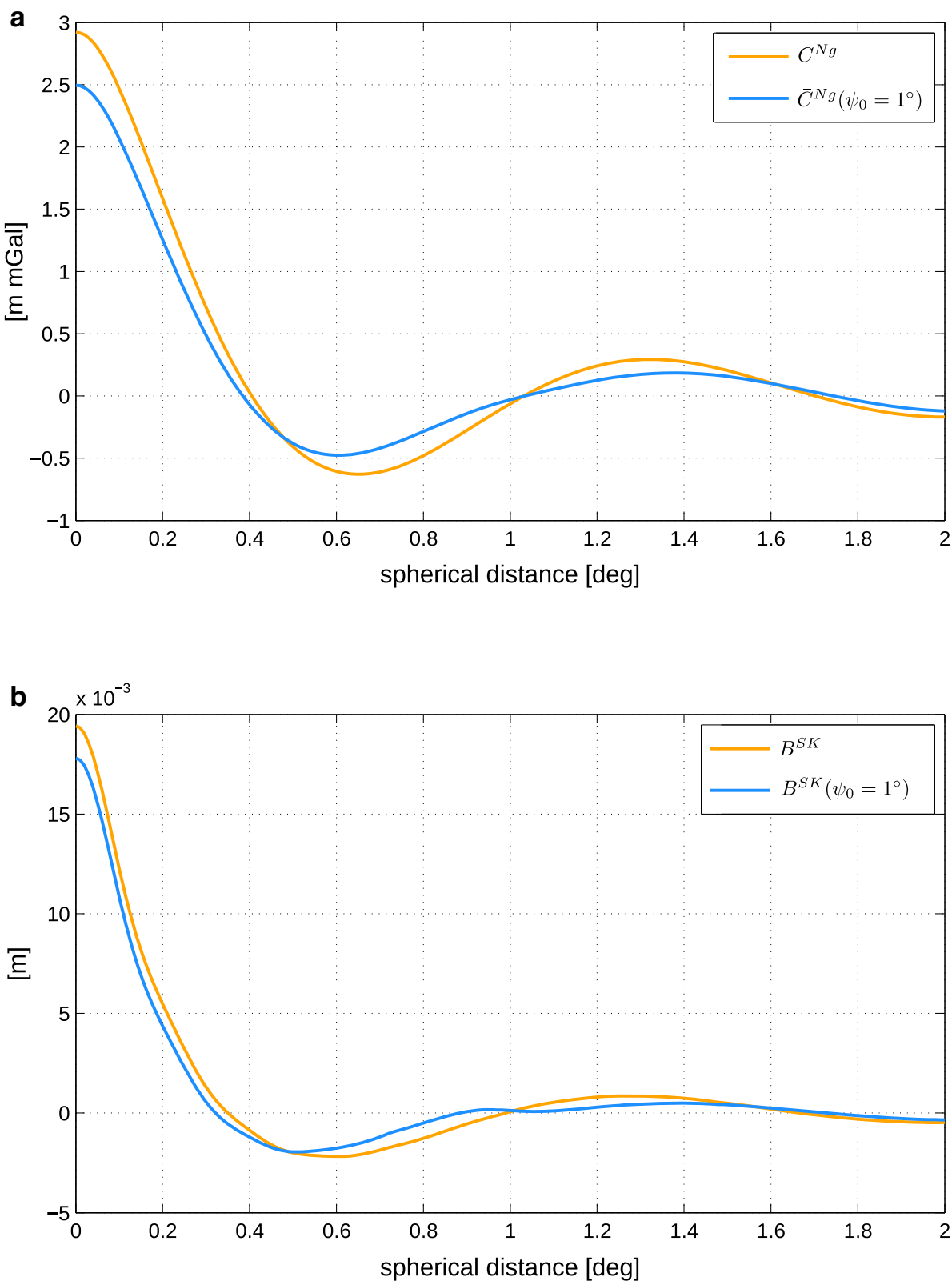

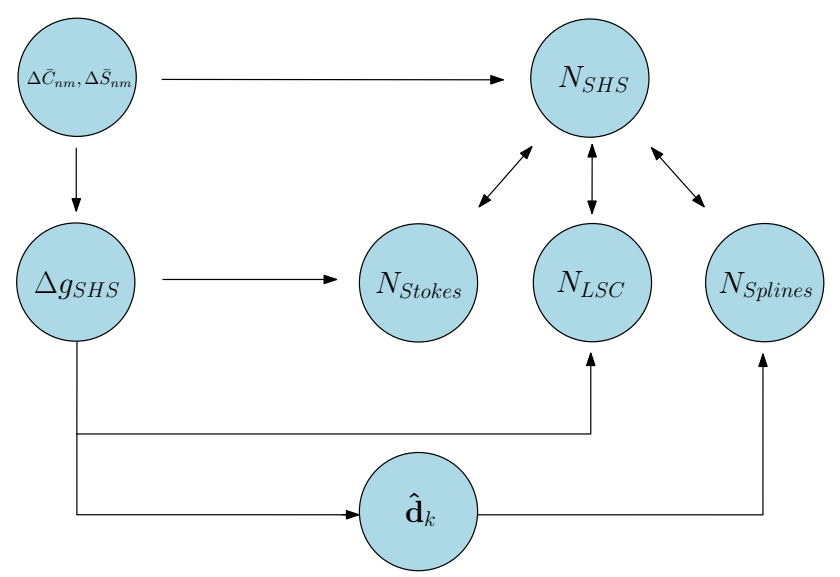

Fig. 2 Schematic of the closed-loop simulation

$$
\begin{aligned}
N(P)= & \sum_{n=251}^{2190} \lambda_{n}^{N}\left[1-\frac{n-1}{2} Q_{n}\left(\psi_{0}\right)\right] \\
& \times \sum_{m=0}^{n}\left[\Delta \bar{C}_{n m} \cos m \lambda+\Delta \bar{S}_{n m} \sin m \lambda\right] \bar{P}_{n m}(\cos \theta)
\end{aligned}
$$

We have considered two regions, namely the North Sea coastal region of East Frisia and the mountainous Alpine region, with, respectively, smooth and moderately rough topography. For practical computational reasons, the input and output grid resolutions (directly related to the number of observations) were set to 5 arcmin (corresponding to the maximum resolution of EGM2008), and the radius of the 
Table 1 Test regions

\begin{tabular}{lll}
\hline & East Frisia & Alpine region \\
\hline Data area & $52^{\circ} \leq \varphi \leq 55^{\circ}$ & $46^{\circ} \leq \varphi \leq 49^{\circ}$ \\
& $5^{\circ} \leq \lambda \leq 10^{\circ}$ & $7.5^{\circ} \leq \lambda \leq 13.5^{\circ}$ \\
Target area & $53^{\circ} \leq \varphi \leq 54^{\circ}$ & $47^{\circ} \leq \varphi \leq 48^{\circ}$ \\
& $6.5^{\circ} \leq \lambda \leq 8.5^{\circ}$ & $9^{\circ} \leq \lambda \leq 12^{\circ}$ \\
\hline
\end{tabular}

spherical integration cap was set to $\psi_{0}=1^{\circ}$. This cap gives theoretical omission errors of approximately 2 and $6 \mathrm{~cm}$ for East Frisia and the Alpine region, respectively (approximated by computing the contribution to $N$ from the area outside the inner zone using EGM2008, with $251 \leq n \leq 2190$ ). However, the omission error is not relevant in our comparison as it is equal for all methods. Around the target areas, we consider enlarged data areas such that each computation point in the target area is surrounded by a $1^{\circ}$ spherical cap containing data, see Table 1.

Geoid heights by Stokes's formula were computed using Eq. (33), implemented according to Eq. (2) using the closed formula for computing the Stokes function (where the function values were set to zero outside the inner zone). Geoid heights by LSC were computed using Eqs. (36) and (37). Equation (37) was developed to degree 2190, corresponding to the maximum resolution of the observations ( $5 \mathrm{arcmin})$. Considering the SKs, dimensionless spline coefficients were estimated using Eq. (16) with Eq. (14). Details regarding the stability of the linear system are shown in Table 2. Subsequently, the spline coefficients were used to compute geoid heights using Eq. (11) with Eq. (39). Similar to LSC, the SKs were developed to degree 2190.

There are several different point grids available for the RBF positions, known as the grid area. Bentel et al. (2013a) explored how different point grids affect RBF modeling results and concluded that differences due to different point grids are very small $\left(\sim 1 \times 10^{-4} \mathrm{~mm}\right.$ to $\left.\sim 0.2 \mathrm{~mm}\right)$, provided the number of grid points is sufficiently large. We have placed the SKs on the equidistributed Reuter grid, where the spherical distance between grid points is almost constant. The number of SKs on the Reuter grid is defined through the control parameter $\gamma$, which is equal to the maximum SH degree $N_{\max }, \gamma=N_{\max }$ (Bentel et al. 2013b). The outer margin widths $w$ of the RBF grid area were determined by the empirical formula of Bentel (2013), where $w \approx 4 \cdot 180^{\circ} /\left(N_{\max }+1\right)$. The number of RBFs will typically be slightly smaller than the number of observations, but approximately equal. In the following, when the equality of the number of RBFs and the number of observations is discussed, it is this approximate equality that is meant.

First, we show that the unmodified LSC and SK formulas applied to the synthetic inner zone data set do not give the same results as Stokes's formula. This is due to the fact that LSC implicitly extrapolates the inner zone data globally (Sect. 4). It is the contribution of the extrapolation that is shown in Fig. 3. For a rigorous evaluation of the extrapolation effect, the comparison of the solutions from LSC and SKs is done with respect to the truncated SH validation geoid (which is practically identical to Stokes's formula). We observe that there is a continuous residual pattern over both data and target areas. In East Frisia, using unmodified LSC (SKs), we get RMS differences of 1.75 and $1.83 \mathrm{~cm}(1.76$ and $1.83 \mathrm{~cm})$ in the data and target areas, respectively. In the Alpine region, using unmodified LSC (SKs), we get RMS differences of 5.73 and $5.18 \mathrm{~cm}(5.74$ and $5.18 \mathrm{~cm})$ in the data and target areas, respectively. The RMS differences for East Frisia and the Alpine region are in the same order of magnitude as the omission (or truncation) error in the respective regions. This shows that the unmodified LSC and SK formulas have taken signal content outside the data area into account when computing the geoid heights, while the Stokes integration has not.

The results of the geoid computations using modified formulas are shown in Table 3 and Figs. 4 and 5. Table 3 shows that all methods agree on the $\mathrm{mm}$ level in the target area, with smaller errors in East Frisia than in the Alpine region. Stokes's formula gives larger errors outside the target area than LSC and the SKs. In the target areas, LSC and SKs show maximum RMS differences of 0.7 and $1.1 \mathrm{~mm}$ (both in the Alpine region), respectively, while RMS differences of

Table 2 Spline representation

\begin{tabular}{llllll}
\hline Resolution (arcmin) & \multicolumn{2}{l}{ East Frisia } & & \multicolumn{2}{l}{ Alpine region } \\
\cline { 2 - 3 } \cline { 5 - 6 } & 5 & 2.5 & & 2.5 \\
\hline No. of observations & 2257 & 8833 & & 2701 & 10,585 \\
No. of SKs & 1842 & 1842 & & 2464 & 2464 \\
cond $(\mathbf{N})$ & $1.2 \times 10^{19}$ & $9.3 \times 10^{18}$ & & $5.7 \times 10^{19}$ & $7.1 \times 10^{19}$ \\
$\alpha_{0}$ & 7115 & $2.8 \times 10^{4}$ & & 6872 & $2.7 \times 10^{4}$ \\
$\alpha$ & 72 & & 6837 & $1.1 \times 10^{4}$ \\
$\operatorname{cond}(\mathbf{N}+\alpha \mathbf{I})$ & $6.0 \times 10^{7}$ & $6.0 \times 10^{7}$ & & $6.0 \times 10^{5}$ & $1.5 \times 10^{6}$ \\
\hline
\end{tabular}



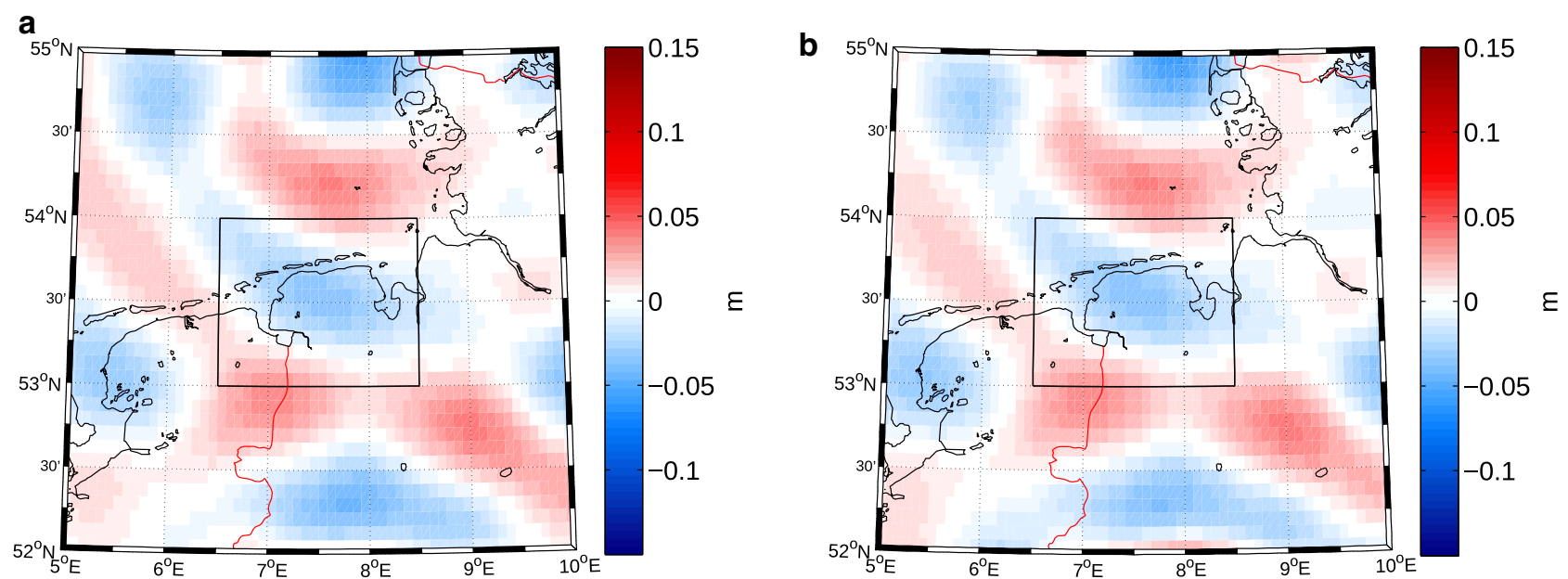

C

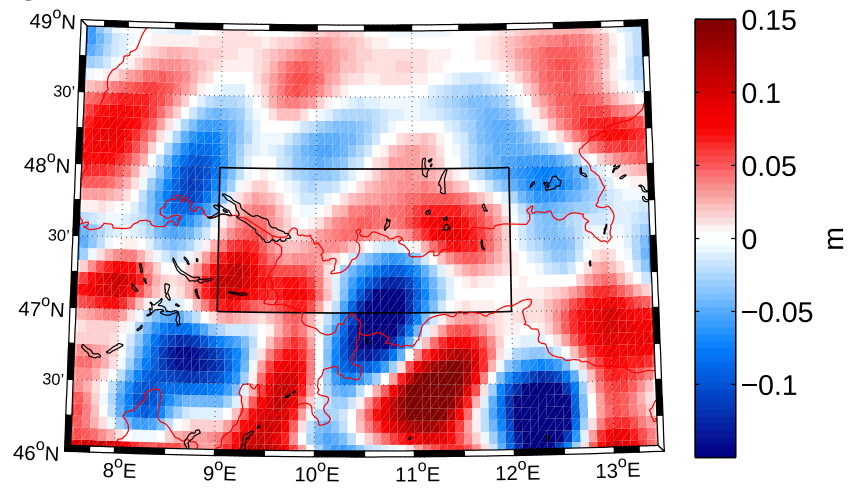

d

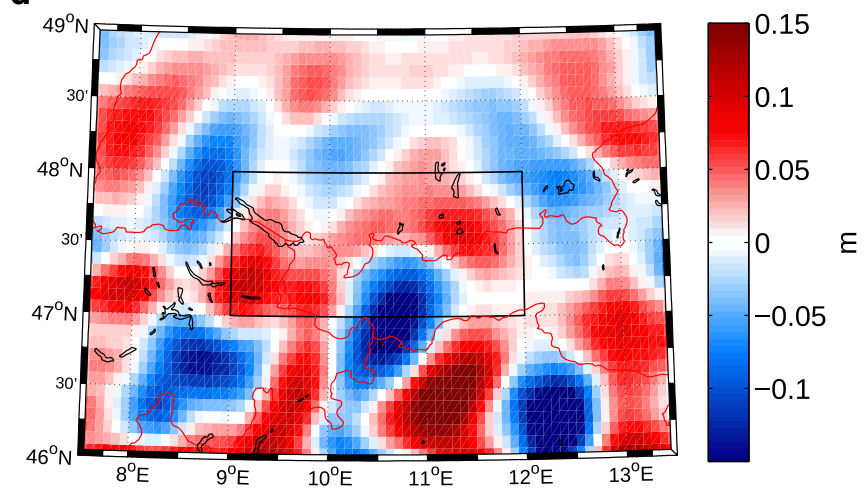

Fig. 3 Deviation of LSC and SKs from the truncated SH validation geoid (simulating Stokes's formula, as given in Eq. (33)) when leaving the covariance function unmodified; a LSC and b SKs in East Frisia, $\mathbf{c}$ LSC and d SKs in the Alpine region

Table 3 Results from the closed-loop simulation, 5 arcmin resolution

\begin{tabular}{|c|c|c|c|c|c|c|c|c|}
\hline & \multicolumn{4}{|c|}{ East Frisia } & \multicolumn{4}{|c|}{ Alpine region } \\
\hline & Max & Min & Mean & RMS & Max & Min & Mean & RMS \\
\hline \multicolumn{9}{|l|}{ Data area } \\
\hline$\Delta g_{\mathrm{SHS}}$ & 11.617 & -6.881 & 0.316 & 3.242 & 46.704 & -63.217 & -0.677 & 12.762 \\
\hline$N_{\mathrm{SHS}}$ & 17.474 & -10.942 & 0.513 & 5.200 & 66.092 & -93.867 & -1.013 & 18.765 \\
\hline$N_{\text {SHS }}-N_{\text {Stokes }}$ & 14.165 & -5.426 & 1.204 & 3.410 & 28.229 & -35.504 & -2.477 & 7.218 \\
\hline$N_{\mathrm{SHS}}-N_{\mathrm{LSC}}$ & 1.880 & -2.594 & 0.006 & 0.644 & 7.548 & -7.952 & -0.007 & 1.354 \\
\hline$N_{\text {SHS }}-N_{\text {Splines }}$ & 2.118 & -3.256 & -0.024 & 0.795 & 6.518 & -6.345 & -0.011 & 1.434 \\
\hline \multicolumn{9}{|l|}{ Target area } \\
\hline$N_{\mathrm{SHS}}$ & 7.297 & -9.076 & 1.870 & 4.241 & 40.613 & -35.447 & -1.669 & 13.678 \\
\hline$N_{\text {SHS }}-N_{\text {Stokes }}$ & 0.419 & -0.638 & 0.082 & 0.173 & 1.087 & -1.598 & -0.087 & 0.588 \\
\hline$N_{\mathrm{SHS}}-N_{\mathrm{LSC}}$ & 0.003 & -0.020 & -0.001 & 0.002 & 0.212 & -0.208 & 0.000 & 0.065 \\
\hline$N_{\text {SHS }}-N_{\text {Splines }}$ & 0.010 & -0.044 & -0.003 & 0.007 & 0.162 & 0.017 & 0.107 & 0.113 \\
\hline
\end{tabular}

Gravity anomalies in $\mathrm{mGal}\left(=10^{-5} \mathrm{~ms}^{-2}\right)$ and geoid heights in $\mathrm{cm}$
$1.7 \mathrm{~mm}$ (East Frisia) and $5.9 \mathrm{~mm}$ (Alpine region) are found using Stokes's formula. Of all three methods, LSC gives the smallest error.
Looking at Figs. 4 and 5 we see that the errors in the target areas are much lower than in their exterior, showing the effect of truncated computation. In particular, LSC and 

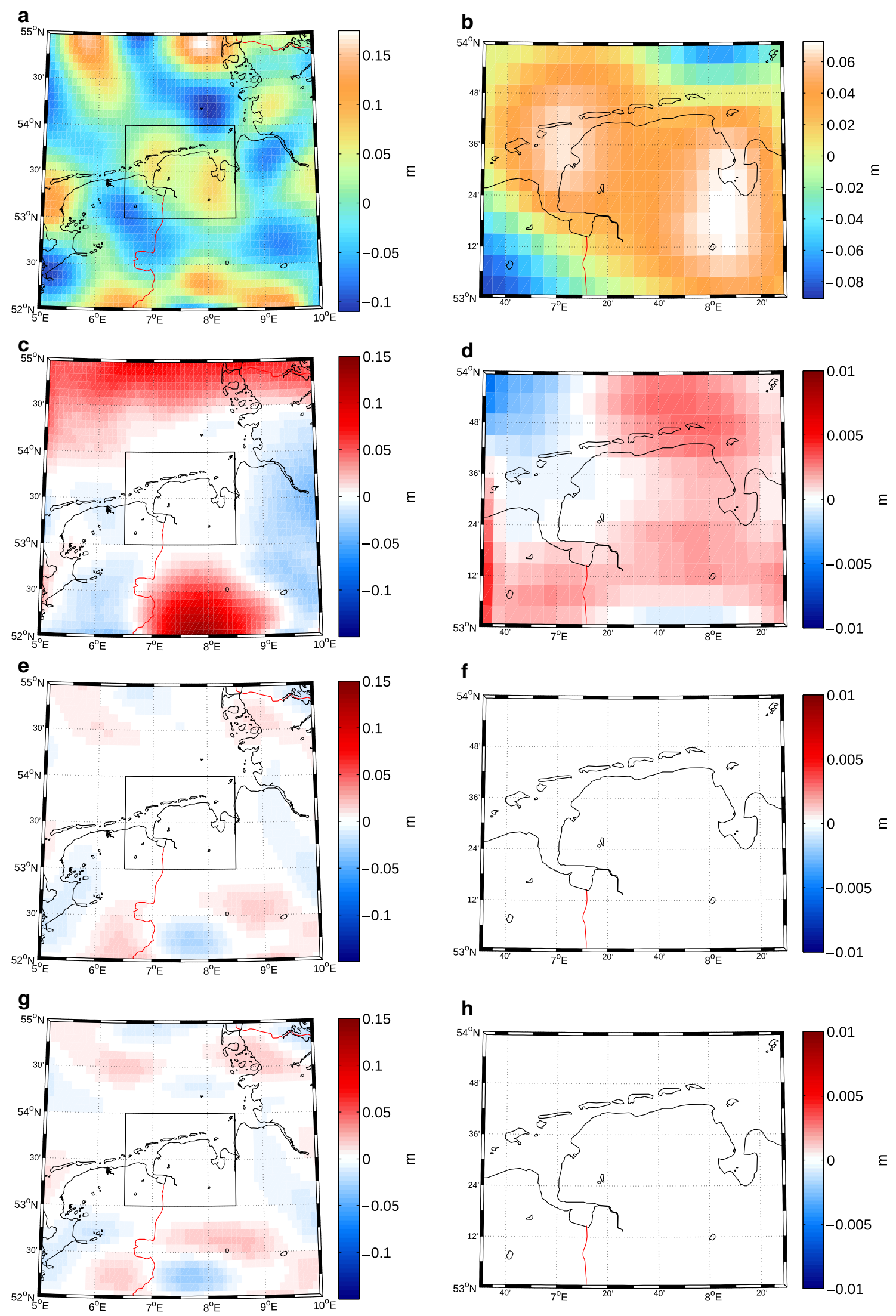

Fig. 4 Results in East Frisia; geoid from SHS in a data and b target areas, error from Stokes's formula in $\mathbf{c}$ data and $\mathbf{d}$ target areas, error from LSC in $\mathbf{e}$ data and $\mathbf{f}$ target areas, and error from SKs in $\mathbf{g}$ data and $\mathbf{h}$ target areas 
a
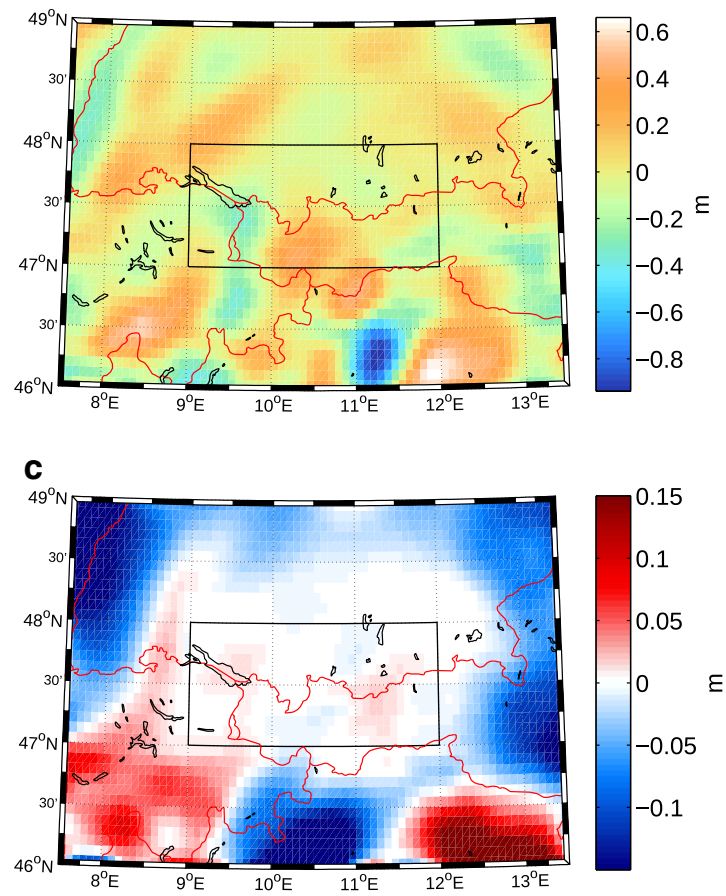

e

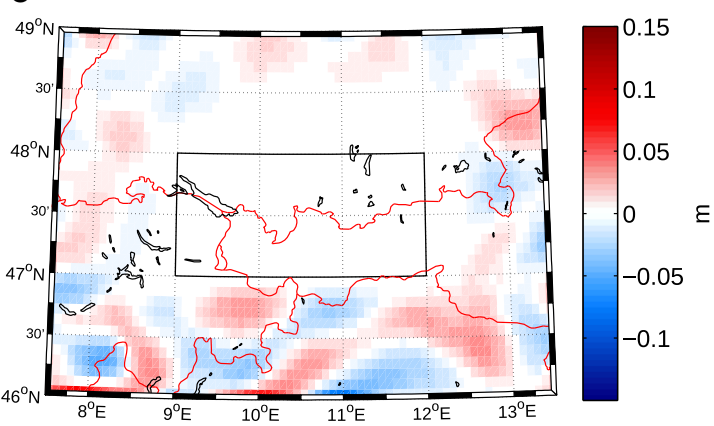

g

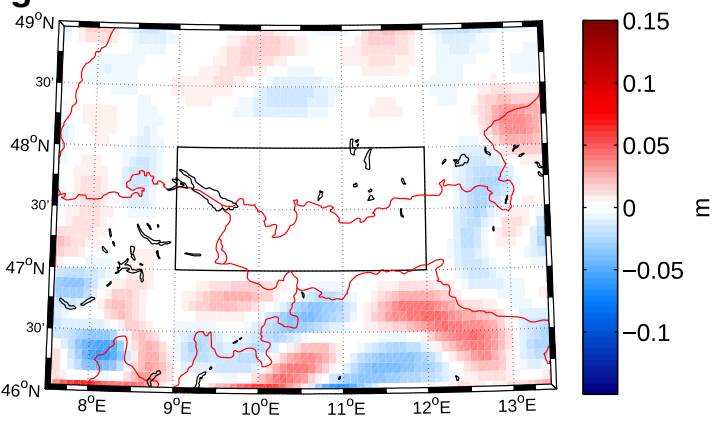

b

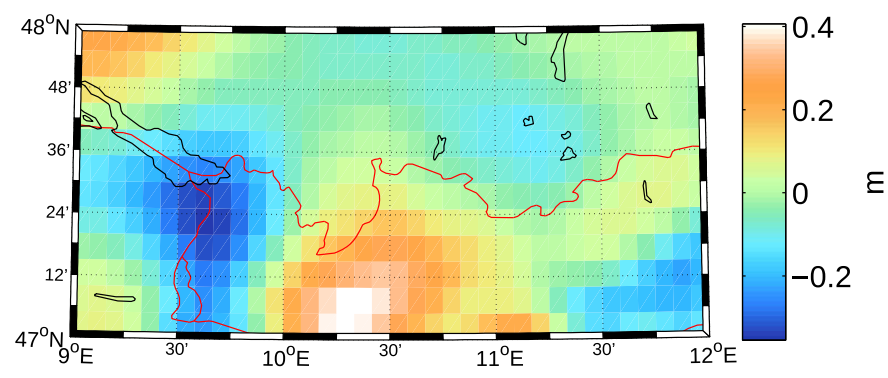

d

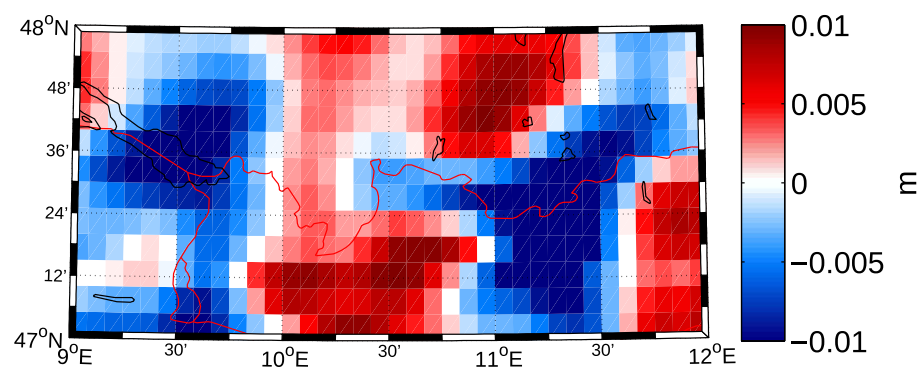

f

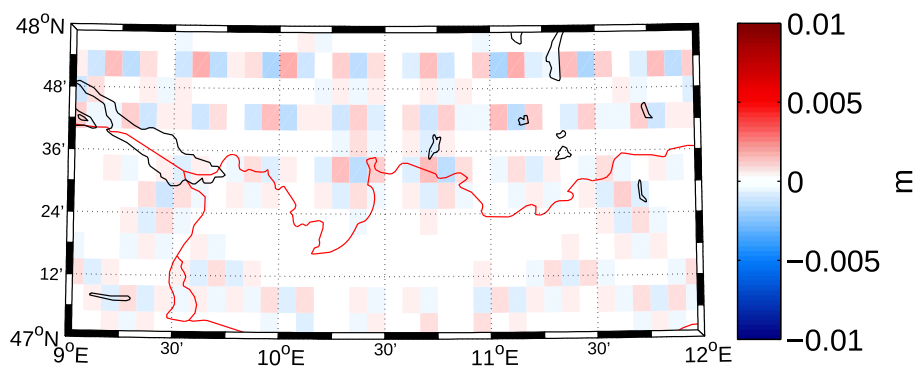

h

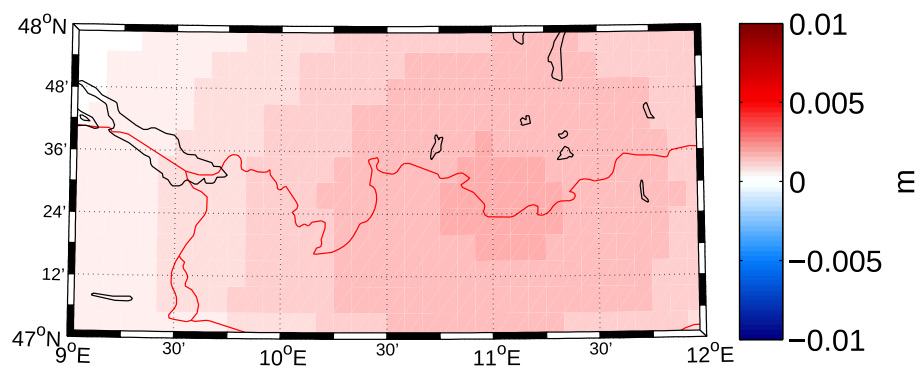

Fig. 5 Results in Alpine region; geoid from SHS in a data and $\mathbf{b}$ target areas, error from Stokes's formula in $\mathbf{c}$ data and $\mathbf{d}$ target areas, error from LSC in e data and $\mathbf{f}$ target areas, and error from SKs in $\mathbf{g}$ data and $\mathbf{h}$ target areas

the SKs show smaller errors in the target area, which was not the case when using the unmodified formulas (Fig. 3). We also note the similarity in the error patterns of LSC and SKs.
The slightly larger RMS differences in Stokes's formula in comparison with LSC/SKs could be owing to the fact that the synthetic observations are point values, which SHS and 
Table 4 Results from the closed-loop simulation, 2.5 arcmin resolution

\begin{tabular}{|c|c|c|c|c|c|c|c|c|}
\hline & \multicolumn{4}{|c|}{ East Frisia } & \multicolumn{4}{|c|}{ Alpine region } \\
\hline & Max & Min & Mean & RMS & Max & Min & Mean & RMS \\
\hline \multicolumn{9}{|l|}{ Data area } \\
\hline$\Delta g_{\mathrm{SHS}}$ & 11.794 & -6.894 & 0.296 & 3.219 & 46.736 & -63.347 & 0.634 & 12.723 \\
\hline$N_{\mathrm{SHS}}$ & 17.536 & -10.942 & 0.481 & 5.158 & 66.515 & -94.385 & -0.955 & 18.729 \\
\hline$N_{\text {SHS }}-N_{\text {Stokes }}$ & 14.422 & -5.894 & 1.032 & 3.273 & 30.577 & -37.650 & -2.107 & 7.056 \\
\hline$N_{\mathrm{SHS}}-N_{\mathrm{LSC}}$ & 3.717 & -3.266 & 0.031 & 0.996 & 12.432 & -10.639 & -0.051 & 2.098 \\
\hline$N_{\text {SHS }}-N_{\text {Splines }}$ & 2.317 & -3.385 & -0.022 & 0.786 & 4.377 & -5.683 & -0.019 & 1.257 \\
\hline \multicolumn{9}{|l|}{ Target area } \\
\hline$N_{\text {SHS }}$ & 7.333 & -9.076 & 2.097 & 4.247 & 41.130 & -35.447 & -1.936 & 13.620 \\
\hline$N_{\text {SHS }}-N_{\text {Stokes }}$ & 0.429 & -0.512 & 0.037 & 0.088 & 0.529 & -0.663 & -0.041 & 0.242 \\
\hline$N_{\mathrm{SHS}}-N_{\mathrm{LSC}}$ & 0.057 & -0.103 & -0.001 & 0.010 & 0.059 & -0.054 & 0.000 & 0.015 \\
\hline$N_{\text {SHS }}-N_{\text {Splines }}$ & -0.002 & -0.040 & -0.004 & 0.006 & 0.110 & 0.024 & 0.079 & 0.082 \\
\hline
\end{tabular}

Gravity anomalies in $\mathrm{mGal}\left(=10^{-5} \mathrm{~ms}^{-2}\right)$ and geoid heights in $\mathrm{cm}$
LSC/SKs expect. However, Stokes integration, while applied point-wise, expects block mean values. To test to what extent the larger RMS differences for Stokes's formula are due to a discretization error, we set up the closed-loop simulation with 2.5 arcmin grid resolution, see Table 4 . Increasing the grid resolution to 2.5 arcmin leaves the LSC and SK results largely unaffected (with maximum improvements in the target areas of $\sim 0.5 \mathrm{~mm}$, and even a slight degradation of the LSC solution in East Frisia), while results by Stokes's formula improve by 0.85 and $3.5 \mathrm{~mm}$ in East Frisia and Alpine region, respectively. Thus, we identify the larger discrepancies using Stokes's formula at the 5 arcmin grid resolution as a discretization error. Furthermore, as there is nothing to gain from increasing the grid resolution of the observations (at 5 arcmin, they are already at their maximum signal resolution), we attribute the discretization error to the Stokes function and not the gravity data. The error is a result of the Stokes function being evaluated only at each grid point of the input data, instead of evaluating its surface integral over the corresponding blocks. Thus, with increased resolution and decreased block size, the function value at the grid nodes gives an increasingly better representation of the surface integral over the grid compartments, and Stokes's formula converges toward SHS.

Finally, we explore how the number of SKs affects the RMS differences (and thus the equivalence to the other methods). In Sect. 3, LSC and SKs were found to be theoretically equal in the continuous case, respectively, in case of an infinitely dense distribution of SKs. In the following, we want to explore how dense the point grid needs to be in practical applications. We repeat the computations for the Alpine region, still using gravity anomalies with 5 arcmin signal resolution given on the 5 arcmin grid, but varying the number of SKs. The regularization parameter was kept constant to make the computations consistent, securing that the

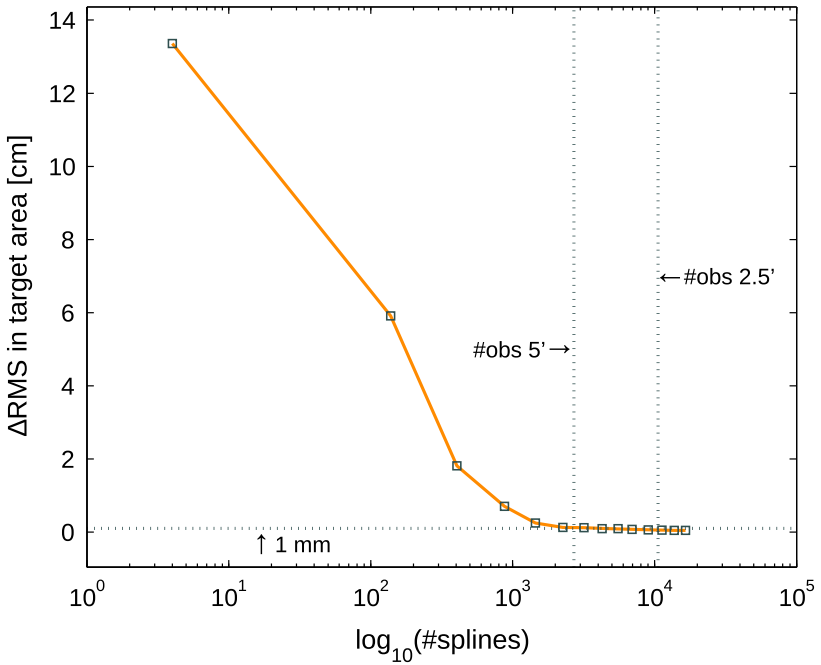

Fig. 6 Difference between SHS and SKs for varying number of SKs in the Alpine target area, with $\alpha=\alpha_{0}=$ const.

only variable in the test is the number of SKs. Again, the RMS differences, or modeling errors, are with respect to the truncated SH validation geoid. If the error is small for a certain number of SKs, we can conclude that the SKs are also similar to LSC, thus providing a measure of how dense the SKs need to be placed in practice. Because we already know that the different methods agree on the mm level, we have chosen $1 \mathrm{~mm}$ as the modeling error threshold.

Figure 6 shows the modeling error as a function of the number of SKs. The vertical dotted lines denote the number of observations corresponding to the 5 arcmin and $2.5 \mathrm{arcmin}$ grid resolutions, while the horizontal dotted line denotes the 1-mm modeling error threshold with respect to SHS. We observe that the error decreases rapidly with increasing number of SKs until it reaches the 1-mm threshold using roughly 
3000 SKs. This corresponds to the number of grid points of the 5 arcmin input grid and, in our case, represents both the number of observations (input grid resolution) and the signal resolution (spectral content). To test which of the two is decisive, we computed solutions using a denser 2.5 arcmin grid while keeping the signal resolution constant at 5 arcmin (leading to a slightly oversampled signal). Based on this input data grid, we performed two modeling runs, where the number of SKs corresponds to either (1) the signal resolution (corresponding to 5 arcmin, see Table 2) or (2) the number of observations (9738 SKs, corresponding to $2.5 \mathrm{arcmin}$ ). Both cases give practically equal results (RMS differences between SK geoid solutions of $1 \mathrm{~mm}$ and $5 \times 10^{-2} \mathrm{~mm}$ in the data and target zones, respectively), suggesting that the SKs converge toward LSC depending on the signal resolution rather than the number of observations. Using more observations increases redundancy and helps to filter out observation noise, but it is not necessary to use an equally increased number of SKs to adequately represent the gravity field.

\section{Summary}

We have reviewed the theoretical equivalence of Stokes's formula, LSC, and SKs in the global case, as well as in regional applications, where Stokes integration is restricted to a spherical cap around the computation point, and no data outside this cap is considered. If LSC is not applied globally, its result will be different from Stokes's formula, because an unwanted extrapolation outside the cap takes place. If the cross-covariance function is modified appropriately, LSC and Stokes's formula are again equal. This has already been shown by de Min (1995). As SKs are equivalent to LSC, they have to be modified correspondingly to give equal results as Stokes's formula.

With a few numerical examples, we have shown that the methods are equal also in practice. Two regions were considered, East Frisia and the Alpine region, with small and large gravity field variations, respectively. At the 5 arcmin resolution, all methods agree within $2 \times 10^{-2} \mathrm{~mm}$ to $5.9 \mathrm{~mm}$ in the target areas, where the largest RMS differences are due to the discretization of Stokes's formula. At the 2.5 arcmin resolution, all methods agree within $6 \times 10^{-2} \mathrm{~mm}$ to $2.4 \mathrm{~mm}$. In general, the remaining discrepancies can be expected due to the varying numerical implementations. For example, Novák et al. (2001) found remaining differences on the mm level between the theoretically equivalent numerical integration and 1D-FFT evaluations of Stokes's formula at the 5 arcmin resolution, which they attribute to the numerical accuracy of their implemented algorithms.

From a theoretical point of view, LSC should give the best results because the covariance function is the only kernel which has the minimum variance property (Moritz 1980).
Indeed, this is confirmed in our numerical examples, where LSC generally gives the smallest error. SKs perform very similar to LSC. From the theoretical point of view, both methods should be identical in the continuous case, i.e., when placing the SKs on an infinitely dense point grid. Our numerical examples show that this limit case does not have to be reached in practice. Rather, it suffices to choose the SK grid spacing according to the signal resolution. This is directly related to the ability of the SKs to achieve an adequate representation of the gravity field. Using less SKs than associated with the signal resolution leads to an inadequate gravity field representation, i.e., the modeling error increases with decreasing number of SKs. Using more SKs than associated with the signal resolution leads to overparametrization. This generally increases the numerical effort (inversion of an unnecessarily large matrix) and may lead to instabilities of the normal equation system. Thus, the SKs are principally independent of the number and distribution of observations. When measuring the numerical effort of SKs and LSC in terms of the size of the matrix that needs to be inverted (normal equation matrix in case of the SKs and the autocovariance matrix of the observations in case of LSC), the effort needed for LSC depends on the number of observations, while the effort needed for the SKs depends on the signal resolution.

In practice, of course, signal and grid resolutions are interrelated. If the distance between adjacent observation points decreases, more small-scale features of the signal can be modeled, which in turn requires a denser grid of SKs. The limit is reached at the point where there are no significant signal variations between the observations. At this point, the number of observations is sufficiently high to represent the full spectral content of the signal. Then, the numerical effort for both SKs and LSC is similar. If the number of observations is higher (which could help filtering out observational noise), the numerical effort increases for LSC, but not for SKs. Furthermore, in reality, the distribution of observations is typically heterogeneous, and placing the SKs on a regular grid will never give optimal results (Wittwer 2009). One possibility to overcome this problem would be to determine the grid spacing for the SKs depending on the resolution necessary to reach a certain accuracy requirement for the final modeling results (e.g., a 1-cm geoid). Another possibility could be to choose a scattered placement of the SKs, where more SKs are used for parts of the data set where the signal is rough, and less in parts where the signal is smoother. Different data-adaptive approaches have been used to generate such a scattered distribution, see, for example, Klees et al. (2008).

To complete the discussion on spacing between and distribution of the SKs, we also stress that the spacing and distribution are not the only variables in the RBF approach, but also the bandwidth (i.e., the maximum degree to which 
the SKs are developed) needs to be adapted according to the signal resolution (Wittwer 2009). This was not explicitly discussed in our numerical examples, because the signal resolution and bandwidth were always set to $N_{\max }=2190$.

Finally, we point out that our aim has not been to decide which regional geoid computation method is the best, as there are advantages and drawbacks to all depending on the data situation. Furthermore, our numerical examples do not represent the most efficient implementations of each method, as they rather aim to compare the outcome of the different methods. Thus, we have not compared the numerical implementations in terms of computation times and limitations. In addition, this work does not present new theory, but is a first attempt to compare the three methods both theoretically and numerically in a unified framework. We have demonstrated that the three methods give equal results in applications, for which a modification of LSC and SKs was necessary. We stress that this modification is not a general necessity when applying the LSC and RBF approaches. However, de Min (1995) points out a few advantages of modifying the covariance function. For example, the modification makes the LSC results less dependent on the validity of the covariance function, as its validity is of most importance for the extrapolated data, which is not considered anymore. Although the modification is not necessary for the application of LSC and RBFs in general, it is critical in direct comparison with Stokes's formula.

Acknowledgements The authors would like to thank R. Rummel and M. Šprlák for helpful comments on an early version of the manuscript. It was further improved by constructive comments from the Editor-in-Chief and three anonymous reviewers, which are gratefully acknowledged. Figures were drafted using the M_Map package, with coastlines and political boundaries from the National Oceanic and Atmospheric Administration and Natural Earth, respectively.

Open Access This article is distributed under the terms of the Creative Commons Attribution 4.0 International License (http://creativecomm ons.org/licenses/by/4.0/), which permits unrestricted use, distribution, and reproduction in any medium, provided you give appropriate credit to the original author(s) and the source, provide a link to the Creative Commons license, and indicate if changes were made.

\section{References}

Bentel K (2013) Regional gravity modeling in spherical radial basis functions - on the role of the basis function and the combination of different observation types. $\mathrm{PhD}$ thesis, Norwegian University of Life Sciences

Bentel K, Schmidt M, Denby CR (2013) Artifacts in regional gravity representations with spherical radial basis functions. J Geod Sci 3:173-187. doi:10.2478/jogs-2013-0029

Bentel K, Schmidt M, Gerlach C (2013b) Different radial basis functions and their applicability for regional gravity field representation on the sphere. Int J Geomath 4:67-96. doi:10.1007/ s13137-012-0046-1
Bouman J (1998) Quality of regularization methods. Tech. Rep. 98.2, DEOS Report, Delft University Press, Delft, The Netherlands

Bucha B, Bezděk A, Sebera J, Janák J (2015) Global and regional gravity field determination from GOCE kinematic orbit by means of spherical radial basis functions. Surv Geophys 36(6):773-801. doi:10. 1007/s10712-015-9344-0

Bucha B, Janák J, Papčo J, Bezděk A (2016) High-resolution regional gravity field modelling in a mountainous area from terrestrial gravity data. Geophys J Int 207(2):949-966. doi:10.1093/gji/ggw311

Denker H (2013) Regional gravity field modeling: theory and practical results. In: Xu G (ed) Sciences of geodesy-II. Springer, Berlin, pp 185-191. doi:10.1007/978-3-642-28000-9_5

de Min E (1995) A comparison of Stokes' numerical integration and collocation, and a new combination technique. Bull Géod 69:223232. doi:10.1007/BF00806734

Eicker A (2008) Gravity field refinement by radial basis functions from in-situ satellite data. $\mathrm{PhD}$ thesis, University of Bonn

Eicker A, Schall J, Kusche J (2014) Regional gravity modelling from spaceborne data: case studies with GOCE. Geophys J Int 196:1431-1440. doi:10.1093/gji/ggt485

Farahani HH, Slobbe DC, Klees R, Seitz K (2016) Impact of accounting for coloured noise in radar altimetry data on a regional quasi-geoid model. J Geod. doi:10.1007/s00190-016-0941-6

Flury J (2006) Short-wavelength spectral properties of the gravity field from a range of regional data sets. J Geod 79(10):624-640. doi:10. 1007/s00190-005-0011-y

Freeden W, Schneider F (1998) An integrated wavelet concept of physical geodesy. J Geod 72(5):259-281. doi:10.1007/s001900050166

Freeden W, Gervens T, Schreiner M (1998) Constructive approximation on the sphere, with applications in geomathematics. Clarendon Press, Oxford

Haagmans R, de Min E, von Gelderen M (1993) Fast evaluation of convolution integrals on the sphere using 1D FFT, and a comparison with existing methods for Stokes's integral. Man Geod 18:227-241

Hansen PC, O'Leary DP (1993) The use of the L-curve in the regularization of discrete ill-posed problems. SIAM J Sci Comput 14:1487-1503. doi:10.1137/0914086

Heiskanen WA, Moritz H (1967) Physical geodesy. W.H. Freeman, San Francisco

Hofmann-Wellenhof B, Moritz H (2006) Physical geodesy, 2nd edn. Springer, Wien

Jekeli C (1978) An investigation of two models for the degree variances of global covariance functions. Tech. Rep. 275, Dept. of Civil and Environmental Engineering and Geodetic Science, Ohio State University, Columbus

Jekeli C (2005) Spline representations of functions on a sphere for geopotential modeling. Tech. Rep. 475, Dept. of Civil and Environmental Engineering and Geodetic Science, Ohio State University, Columbus

Klees R, Tenzer R, Prutkin I, Wittwer T (2008) A data-driven approach to local gravity field modelling using spherical radial basis functions. J Geod 82:457-471. doi:10.1007/s00190-007-0196-3

Krarup T (1969) A contribution to the mathematical foundation of physical geodesy. Tech. Rep. 44, Danish Geodetic Institute, Copenhagen

Kusche J (2002) Inverse Probleme bei der Gravitationsfeldbestimmung mittels SST- und SGG-Satellitenmissionen. Habilitation thesis, Deutsche Geodätische Kommission Reihe C, Nr. 548

Lieb V, Schmidt M, Dettmering D, Börger K (2016) Combination of various observation techniques for regional modeling of the gravity field. J Geophys Res Solid Earth 121:3825-3845. doi:10.1002/ 2015JB012586

Liu Q, Sideris MG (2003) Wavelet evaluation of the Stokes and Vening Meinesz integrals. J Geod 77(5):345-356. doi:10.1007/ s00190-003-0333-6

Moritz H (1980) Advanced physical geodesy. Wichmann, Karlsruhe 
Moritz H, Sünkel H (eds) (1978) Approximation methods in geodesy. Wichmann, Karlsruhe

Naeimi M (2013) Inversion of satellite gravity data using spherical radial base functions. PhD thesis, Deutsche Geodätische Kommission Reihe C, Nr. 711

Naeimi M, Flury J, Brieden P (2015) On the regularization of regional gravity field solutions in spherical radial base functions. Geophys J Int 202:1041-1053. doi:10.1093/gji/ggv210

Nerem RS, Jekeli C, Kaula WM (1995) Gravity field determination and characteristics: retrospective and prospective. J Geophys Res Solid Earth 100:15,053-15,074. doi:10.1029/94JB03257

Novák P, Vaníček P, Véronneau M, Holmes S, Featherstone W (2001) On the accuracy of modified Stokes's integration in high-frequency gravimetric geoid determination. J Geod 74(9):644-654. doi:10. 1007/s001900000126

Paul M (1973) A method of evaluating the truncation error coefficients for geoidal height. Bull Géod 47:413-425

Pavlis NK, Holmes SA, Kenyon SC, Factor JK (2012) The development and evaluation of the Earth Gravitational Model 2008 (EGM2008). J Geophys Res Solid Earth 117(B04):406. doi:10. 1029/2011JB008916

Pock C, Mayer-Gürr T, Kühtreiber N (2014) Consistent combination of satellite and terrestrial gravity field observations in regional geoid modeling: a case study for Austria. In: Marti U (ed) Gravity, Geoid and height systems: proceedings of the IAG symposium GGHS2012, October 9-12, 2012. Springer, Berlin, pp 151-156. doi:10.1007/978-3-319-10837-7_19

Rexer M, Hirt C (2015) Spectral analysis of the Earth's topographic potential via 2D-DFT: a new data-based degree variance model to degree 90,000. J Geod 89:887-909. doi:10.1007/ s00190-015-0822-4

Roland M (2005) Untersuchungen zur Kombination terrestrischer Schweredaten und aktueller globaler Schwerefeldmodelle. $\mathrm{PhD}$ thesis, Wissenschaftliche Arbeiten der Fachrichtung Vermessungswesen der Universität Hannover, Nr. 254

Roland M, Denker H (2005) Stokes Integration versus Wavelet Techniques for Regional Geoid Modelling. In: Sansò F (ed) A Window on the future of geodesy: proceedings of the international association of geodesy IAG general assembly Sapporo, Japan June 30-July 11, 2003. Springer, Berlin Heidelberg, pp 368-373

Rummel R (1982) Gravity parameter estimation from large and densely spaced homogeneous data sets. Boll Geod Sci Aff 41(2):149-160

Rummel R, Schwarz KP, Gerstl M (1979) Least squares collocation and regularization. Bull Géod 53:343-361. doi:10.1007/BF02522276
Sansò F, Sideris MG (eds) (2013) Geoid determination: theory and methods. Lecture Notes in Earth System Sciences. Springer, Berlin

Schmidt M (2001) Grundprinzipien der Wavelet-Analyse und Anwendungen in der Geodäsie. Shaker Verlag, Aachen

Schmidt M, Martínez W, Florez J (2002) General scheme for the computation of regional geoid undulations using spherical wavelets. In: Drewes H, Dodson AH, Fortes LPS, Sánchez L, Sandoval P (eds) Vertical reference systems: IAG symposium Cartagena, Colombia, February 20-23, 2001. Springer, Berlin Heidelberg, pp 209-214

Schmidt M, Fengler M, Mayer-Gürr T, Eicker A, Kusche J, Sánchez L, Han SC (2007) Regional gravity modeling in terms of spherical base functions. J Geod 81:17-38. doi:10.1007/ s00190-006-0101-5

Schmidt M, Gerlach C, Bouman J, Bentel K, Dettmering D, Eicker A, Herceg M, Kusche J, Lieb V, Schall J, Tscherning CC (2015) Results from IAG's Joint Study Group JSG0.3 on the Comparison of Current Methodologies in Regional Gravity Field Modelling. Poster presented at the 26th International Union of Geodesy and Geophysics General Assembly, Prague, Czech Republic, June 22July 2

Stokes GG (1849) On the variation of gravity on the surface of the Earth. Trans Camb Philos Soc 8:672-695

Tenzer R, Klees R (2008) The choice of the spherical radial basis functions in local gravity field modeling. Stud Geophys Geod 52(3):287-304. doi:10.1007/s11200-008-0022-2

Tikhonov AN (1963) Regularization of incorrectly posed problems. Sov Math Dokl 4:1624-1627

Torge W, Müller J (2012) Geodesy, 4th edn. De Gruyter, Berlin

Tscherning CC (1981) Comparison of some methods for the detailed representation of the Earth's gravity field. Rev Geophys 19:213221. doi:10.1029/RG019i001p00213

Tscherning CC (1999) Construction of anisotropic covariance functions using Riesz-representers. J Geod 73(6):332-336. doi:10. $1007 / \mathrm{s} 001900050250$

Tscherning CC, Rapp RH (1974) Closed covariance expressions for gravity anomalies, geoid undulations and deflections of the vertical implied by anomaly degree variance models. Tech. Rep. 208, Dept. of Geodetic Science and Surveying, Ohio State University, Columbus

Vaníček P, Krakiwsky EJ (1986) Geodesy: the concepts, 2nd edn. North Holland, Amsterdam

Wittwer T (2009) Regional gravity field modelling with radial basis functions. PhD thesis, Technical University of Delft 